\title{
HISTORIA
}

\section{SITUACIÓN DEL PREVENTORIO DE TUBERCULOSOS DE AGUAS DE BUSOT; EXPERIENCIA PERSONAL}

\author{
Rubio López, S*.; Candela Ferri, J*.; Miró Batlle, S*.; Pozo Cisneros, L*. \\ *Alumnos E.U.Enfermería, Alicante. \\ Gabaldón Bravo, E. M., Profesora Titular E.U.E. Alicante
}

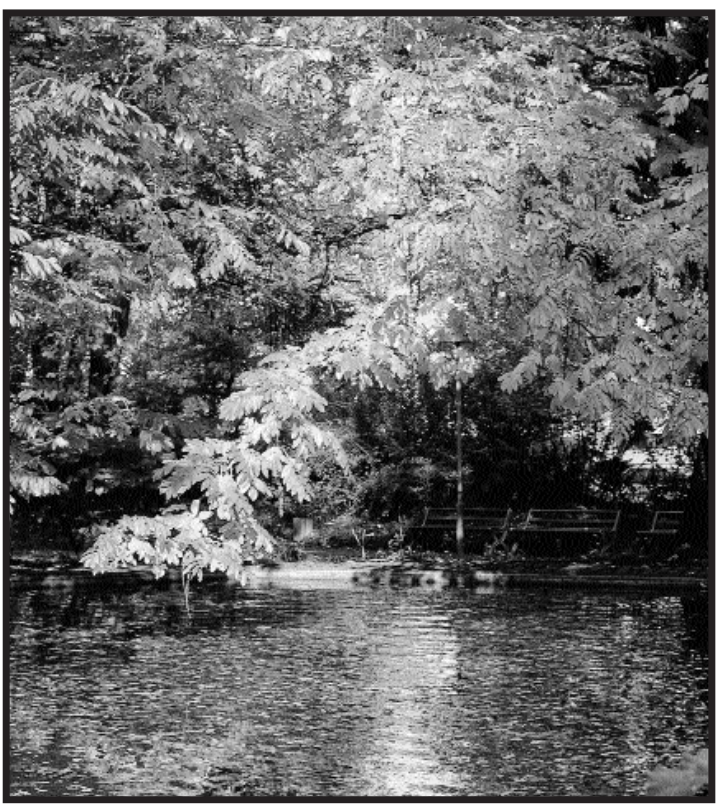

TUBERCULOSIS HOSPITAL IN AGUAS DE BUSOT: A PERSONAL EXPERIENCE

\section{ABSTRACT}

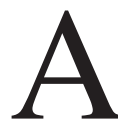
special site with an important historical background is analised, considered here as a reflection of the evolution of the nursing profession with the aim of studying and caring for our past, to improve our present and to create a better future.

Key words:

Historical background, tuberculosis hospital, nursing, tuberculosis, nursing records evolution.

\section{RESUMEN}

71 artículo trata tanto de un análisis sobre un lugar concreto, con gran bagaje histórico, como una reflexión sobre la evolución de la profesión enfermera. Un ánimo al estudio y cuidado de nuestro pasado, para mejorar el presente $\mathrm{y}$ crear un futuro mejor.

\section{Palabras clave:}

Estudio histórico, sanatorio tuberculoso, enfermería, tuberculosis, evolución de registros enfermeros.

\section{INTRODUCCIÓN}

Sin duda la tuberculosis es una enfermedad que acompaña al hombre desde la prehistoria, de hecho, existen referencias escritas desde Egipto, Babilonia y China.

Durante la Revolución Industrial se produjo una exacerbación de esta enfermedad, que fue considerada endémica durante la Edad Media, siendo la productora del $30 \%$ de las muertes del momento.

Pero, centrémonos más en la atención que se prestaba en España al problema de la gran proliferación de casos ya entrado el siglo XIX y principios del XX.

La principal medida (tomada tras la instauración de la II República) fue construir en varios puntos del territorio español Dispensarios y Preventorios antituberculosos y Sanatorios Especializados (también para niños), que en total eran cinco.

Adentrándonos aún más en el tema de los Preventorios, encontramos en Aigües (Municipio 
de la provincia de Alicante), unas instalaciones que sirvieron con este fin hasta hace unos 20 años.

Su ubicación es perfecta para el fin que sirvió, ya que se encuentra en la falda del monte Cabeçó d'Or, a $501 \mathrm{~m}$. sobre el nivel del mar y a solo $5 \mathrm{~km}$. de la costa, ofreciendo un clima saludable.

En la documentación estudiada, no se encuentran datos sobre cuando empezó la actividad como baños de este establecimiento, pero se podría remontar a la época árabe e incluso al Imperio Romano.

Encontramos que en 1816 la Marquesa de Bosch, Condesa de Torrellano, adquirió estos baños para convertirlos en un Balneario Aristocrático al que llamó Hotel Miramar. Este hotel estaba acondicionado para albergar con gran lujo a 250 personas. En 1844, los Baños Viejos, como pasaron a llamarse, fueron adquiridos por el Conde de Casas Rojas, el cual amplió el recinto en torno al edificio principal con 33 casitas para el alojamiento de familias adineradas.

La fama que adquirieron los balos y sus aguas hicieron que en 1936 fuera habilitado para que se trasladase allí el Preventorio Infantil de Tuberculosos del Estado, con el tiempo pasó a especializarse en enfermedades pulmonares y siguió utilizándose con fin sanitario hasta su cierre que podemos situar entre 1970 y 1980.

Por otra parte, y siguiendo a J. Siles, sabemos que el papel de las enfermeras en la lucha antituberculosa, se dividía en dos ramas:

- Las enfermeras dedicadas a la visita domiciliaria, educación, información a pacientes y familiares e investigación, junto con los practicantes que se dedicaban a técnicas concretas (como cirugías menores) y

- Las enfermeras que trabajaban en los sanatorios especializados, en los que se dedicaban a atendes las necesidades básicas, la alimentación, la higiene y la seguridad. Utilización de registros y fichas de ingreso, para identificar mejor a los pacientes y la marcación de la ropa y los enseres de cada grupo en los que se dividían los Sanatorios/ Preventorios.

Partiendo de nuestro interés por conocer un poco más de la historia de nuestra profesión y conociendo la existencia de este edificio, nos planteamos su visita.
En la actualidad, las instalaciones pertenecen a una empresa privada, pero están accesibles a la visita y recogida de documentos y archivos médicos supuestamente confidenciales pero abandonados por el recinto.

En definitiva, lo que pretendemos con este trabajo es comprobar las siguientes hipótesis:

- Los hospitales desde una perspectiva histórica, son una fuente de datos primario excelente para el estudio de la historia de la enfermería, y

- Existe una continuidad no proclive a los cambios en la realización de la labor del profesional de enfermería, que se ve reflejada en la evolución de la calidad y tipo de registros.

\section{MATERIAL Y MÉTODOS}

En cuanto al material, éste se obtuvo a partir de la visita realizada al antiguo sanatorio tuberculoso de Aigües, y previamente a esta visita fuimos al ayuntamiento de este municipio. En dicho ayuntamiento se nos informó de la situación actual privada del Preventorio; nos comunicaron que la información podía ser obtenida en la Diputación de Alicante. Por ello al día siguiente nos desplazamos allí para lograr obtener datos necesarios para continuar con nuestro estudio. También nos sirvió de gran ayuda en la recopilación de la información, la Biblioteca de la Fundación Juan Gil-Albert, próxima a la Diputación de Alicante.

Se decidió realizar la recopilación de dos tipos de material:

Las anotaciones de campo realizadas durante la visita al preventorio de cada uno de los miembros del grupo.

La documentación obtenida tanto de gestión como propia de las historias de los pacientes que se pudo encontrar durante la visita y que resultará de interés histórico-social.

El método empleado en ambos casos fue durante la visita al centro donde se realizaron varias fotografías complementarias a los dos tipos de material. Se recopilaron numerosos papeles esparcidos por el edificio, entre ellos había gráficas de constantes y valoraciones. La mayoría de los documentos se encontraban en la farmacia, aunque no podemos verificar que ese fuera el lugar donde estaban situados cuando funcionaba el Hospital. 
Con posterioridad a la visita todos estos documentos fueron catalogados mediante un orden nominal, describiendo su estado actual y la cantidad de documentos utilizados con el mismo fin (documentos de gestión: administración de medicación, pedidos y avisos de enfermedades de carácter obligatorio; documentos de historia del paciente: registros, gráficas y administración de medicación; y finalmente otros) y las fotografías también se clasificaron en un catálogo del local y adyacentes con una breve descripción. Tanto de los documentos como de las fotografías se ha realizado una selección de las más relevantes para la presentación de este trabajo.

En cuanto a las anotaciones de campo estas se sometieron a un análisis comparativo partiendo de las indicaciones de Manuel Amezcua para la realización del diario de campo.

\section{RESULTADOS}

En cuanto al análisis comparativo de las anotaciones se producen grandes coincidencias en cuanto a las emociones experimentadas durante la visita por parte de los miembros del grupo:

1- De temor, ya que el edificio en la actualidad presenta un aspecto misterioso hasta el punto de ser utilizado para el ocultismo y la parapsicología: “ ...Puedo entender, por otra parte, la curiosidad que despierta a la gente que estudia sucesos paranormales el balneario, porque estando allí, todos los sonidos te inspiran algo tenebroso o simplemente les intentas buscar un origen oscuro que no es el real...", “ ...Otra cosa que también es fácil imaginarse es el sufrimiento de los pacientes mas graves, y las toses profundas y amargas; yo creo que esa idea es la que empuja a los ocultistas a elegirlo como lugar de sus ritos"...

2- De curiosidad, coincidiendo en el hecho de que este trabajo incita a profundizar en el mejor conocimiento de la historia de la profesión: “ ....No es difícil mirar hacia el fondo de un pasillo y tratar de imaginar como sería una mañana o un día normal con las enfermeras vestidas de uniforme y con la cofia....". “... A medida que íbamos descubriendo nuevas casas, cuartos y dormitorios, las preguntas surgían solas:¿ qué dieta seguían?, ¿como se organizaban el trabajo las enfermeras?, ¿qué procedimientos se seguían cuando fallecía un paciente?...". ...Era un bombardeo continuo de preguntas que me hacia a mi misma, porque para mi era como si fuera parte de mi propio pasado.

Ya basándonos en el catálogo elaborado se obtuvieron los siguientes resultados en las categorías que se señalan a continuación:

\section{1- GESTIÓN: 1.1-DIETAS:}

Consta de 5 documentos encontrados en la farmacia, tres de los cuales datan en los años 1966 y 67 , dos escritos a máquina y uno a mano. Indican el menú de un determinado día y el número de plazas. El cuarto y quinto documentos son más antiguos y su estado de deterioro es mayor. Datan en 1938, indicando el numero de dieta y el piso, en total habiendo tres pisos, y aproximadamente 231 plazas.

\section{2- MEDICACIÓN:}

Hemos obtenido dos envases de fármacos diferentes, uno en envase de cartón muy deteriorado y llamado ISONIACIDA, y el otro en envase de cristal muy bien conservado y llamado UROMIL. Además tenemos un prospecto de un producto llamado BABEURRE PAIDO, que parece ser de leche en polvo, indicado para trastornos gastrointestinales. También una ficha de un pedido a farmacia sin cumplimentar; por supuesto se encontraban en la farmacia. Añadimos dos pedidos cumplimentados.

\section{3- ESTADÍSTICA DE MORBILIDAD:}

En ellas se indica la provincia y el ayuntamiento y la relación de los enfermos entrados durante un determinado mes. Un solo documento, sin rellenar y en un estado aceptable de deterioro.

\section{4-PEDIDOS:}

Se trata de un solo documento (pedido de medicamentos) encontrado en la farmacia. Data de mayo de 1965 e indica el género, la cantidad y el precio. En la parte inferior de la hoja firma el Jefe Administrativo y El Peticiario.

\section{5-AVISOS DE CONTAGIOS ENTRE LA POBLACIÓ INFANTIL:}

2 documentos encontrados en la farmacia, con estado avanzado de deterioro, escritos a máquina 
en los cuales firma el director del preventorio. Los dos van dirigidos al Ilmo. Sr. Jefe Provincial de Sanidad de Alicante. En uno de ellos se comunica la aparición de cinco casos de varicela entre la población infantil y el otro informa de los niños vacunados contra la poliomielitis.

\section{2- HISTORIA DEL PACIENTE}

\section{1- PLAN DE CUIDADOS:}

Se trata de una hoja de valoración de enfermería, recogida en la farmacia del Preventorio, se encuentra en buen estado, sin deteriorar; sin embargo está sin cumplimentar. Desgraciadamente no hemos conseguido una rellenada con datos de un posible paciente; solo tenemos dos documentos del mismo tipo. Sigue una estructura similar a la actual, con valoración de antecedentes y actuales, condiciones higiénicas de la vivienda y posición social de la familia, además de seguimiento, exploración y valoración al alta.

\section{2-INGRESOS:}

Es un documento en el cual reclaman el ingreso de un paciente, encontrado nuevamente en la farmacia y en un estado relativamente bueno. Tenemos 6 documentos del mismo tipo datados en distintas fechas, que van del 1937 al 1966. Recoge un reclamo con el nombre del paciente, plazo para ingresar, condición de cama gratuita o pagando, el sello del preventorio y las firmas del secretario general y del director del preventorio.

\section{3- GRÁFICAS:}

Documento en el cual se recogen las constantes vitales de los pacientes, encontrado en la farmacia y con un muy mal estado, encontrándose muy deteriorado. Poseemos dos documentos del mismo tipo y en este caso están rellenados los dos. Primero tiene un registro a modo de gráfico del pulso, respiración y temperatura, en el cual observamos que sólo se registraba el pulso y la temperatura. Luego se registraban: tos expectoración, deposiciones, el régimen de cura y otras observaciones.

\section{4- ALTAS:}

Documento en el que se informa a la familia de que su hijo puede ser recogido al dársele el alta, y en caso contrario sería recogido por la Junta de
Protección de la infancia de Alicante. Se encontró nuevamente en la farmacia en un estado aceptable, poco deteriorado. Figura la firma del director y en este caso no hay sello.

\section{5- PARTES DE INFORMACIÓN:}

En ellos se explicaba a los padres o familiares el estado de salud del niño y la imposibilidad de visitarles. Añade otras normas a seguir, entre las cuales prohíben mandarles comida. Encontrados en la farmacia, el estado es variable, algunos están en muy buen estado y otros se encuentran muy deteriorados. Poseemos cuatro diferentes.

\section{6-PETICIONES:}

En ellas se pide alargar la instancia del paciente por parte del director y dirigido a la familia. Hay dos diferentes y en muy mal estado, muy deteriorados.

\section{7-REGISTROS}

\subsection{1-ODONTOLOGÍA:}

Se trata de una ficha en la que aparecen los datos personales del paciente en la parte superior, un esquema de las piezas dentales, indicándose las piezas que faltan; y una valoración compuesta por el estado de los dientes con tres respuestas predeterminadas, al igual que el uso del cepillo, estado de la encía, número del caries y número de abcesos y raíces. Encontrado en la farmacia en buen estado, y tres de los siete están cumplimentados con el nombre del paciente y la edad, faltando la fecha.

\subsection{2- FICHAS PERSONALES:}

14 tarjetas encontradas en la farmacia en buen estado, la mayoría. En ellas se indica el diagnóstico, la edad, la fecha de ingreso, el nombre, el número de historia clínica y la fecha del alta en este orden. El color azul era para el sexo femenino y el azul para el masculino.

\subsection{3-HOJA DE RADIOSCOPIA}

Documento en el cual se observa el nombre de la paciente (ya que está debidamente cumplimentado), el numero de historia, el estado del tórax y por último la fecha (no puesta en este caso). La encontramos en la farmacia y se observan en ella los bordes superiores quemados recientemente. 


\section{8-VALORACIONES:}

2 valoraciones del tórax, una cumplimentada y la otra sin cumplimentar, explicando la sintomatología general en años seguidos del mismo paciente. También hay una hoja de neumotórax de valoración mensual. Todo ello en buen estado, excepto la cumplimentada y encontrados en la farmacia.

\section{9-LABORATORIO:}

2 hojas de laboratorio en las que figuran análisis de sangre, esputos orina y reacción a la tuberculina, con posibilidad de realizarse otras pruebas. Ambas sin cumplimentar y con un grado medio de deterioro.

\section{3-OTROS}

\section{1-CONTRATOS:}

Se trata de tres documentos rellenados totalmente. En ellos se observa el nombre y demás datos del contratado, a cargo del patronato nacional de tuberculosos, en el que consta como cocinero. También se indican las personas en ese momento a su cargo. Los tres contratos son del mismo año y distinto mes. Estos papeles a diferencia de los otros los encontramos en la casa situada al lado de la capilla, en frente de la puerta principal del hospital. Son unos de los impresos que en mejor estado están.

\section{2-CERTIFICADOS:}

Documento del cual no conocemos mucho, indica la relación de certificados que se cursan por conducto de la carteria rural en la localidad de Aguas de Busot en agosto de 1957. Fue encontrado en la farmacia, lleva impresos dos sellos, uno del ayuntamiento y otro del sanatorio. En muy buen estado de conservación.

\section{3-PARTITURA:}

Partitura encontrada en los alrededores de la capilla. Alto grado de deterioro. Parece ser que está impreso por la Casa Editorial de Música "BOILEAU" en Barcelona.

\section{4-LIBRO DE INDICACIONES MÉDICAS:}

Encontrado en la casa de enfermeras, con un estado medio de deterioro. Contiene consejos en administración de medicación y demás tareas. La revista es de 1961.

\section{5-CARTA PERSONAL:}

Está muy deteriorada, parece ser del año 1956, creemos que iba dirigida al sacerdote por lo poco que se puede leer en ella.

\section{6-PÁGINAS PERTENECIENTES A UN LIBRO DE QUÍMICA Y FÍSICA:}

Sólo se puede observar las páginas parcialmente, por lo tanto también están muy demacradas.

\section{7-B.O.E:}

Página perteneciente al boletín oficial del estado. Es del 19 de octubre de 1941. Buen estado de conservación.

\section{8-PERIÓDICO:}

Data del 24 de julio de 1971. Es el diario del municipio de Benidorm. Encontrado en la puerta de la casa de los médicos.

\section{CONCLUSIONES:}

La visita al sanatorio resulta ser de gran riqueza como fuente primaria, a pesar de su estado de deterioro y espolio, tanto en si mismo como por la documentación hallada en él. Ésta documentación nos habla de cómo se desarrollaba la gestión del centro, a través de ver las hojas de pedido, las hojas de dietas, donde se reflejan los menús, y la cantidad de camas ocupadas. También podemos ver, referente a la historia del paciente, la semejanza de los registros que contienen con los que en la actualidad se manejan en los hospitales, sobre todo en las gráficas de constantes, la valoración,... En ningún momento se ha podido constatar que estos documentos fueran cumplimentados por profesionales de enfermería, aunque sabemos que entre las funciones que desarrollaban estos profesionales en éste hospital, se encontraba ésta, la cumplimentación de determinados documentos dentro de la historia del paciente, igual que en la actualidad.

En cuanto a otros documentos empleados, destacamos por su interés, ya que nos habla de la vida cotidiana del centro, el libro de indicaciones médicas, encontrado en la casa de las enfermeras con un estado medio de deterioro; que contiene consejos 
en administración y medicación y demás tareas, los contratos, las partituras del coro, cartas personales de recomendación para el ingreso del paciente.

Encontramos todos los documentos catalogados de tanta valía para la historia de nuestra profesión que no comprendemos que su situación, en el momento de su hallazgo fuera tan deplorable y planificamos su donación al fondo de archivos de la Fundación JUAN GIL-ALBERT en próximas fechas.

A través del análisis de las anotaciones de campo podemos concluir la importancia que esta visita ha tenido para nuestra formación como profesionales, ya que supone un enriquecimiento de la visión histórica de la enfermería.

Nos ayudó a comprender de donde venimos y cuales eran nuestros predecesores en la profesión, donde se desarrollaba su trabajo,... La falta de identificación de los profesionales independientemente de su profesión en todos los documentos, es destacable como dato histórico porque nos hace invisibles y nos hace cuestionarnos si en la actualidad ocurre lo mismo.

Concluimos así la importancia de identificarnos en todo lo que escribimos como profesionales de enfermería para hacernos visibles.

Como grupo, tenemos la intención de profundizar en el estudio del material recopilado, ya que como hemos dicho con anterioridad lo consideramos una fuente primaria excelente para el estudio de la historia de nuestra profesión.

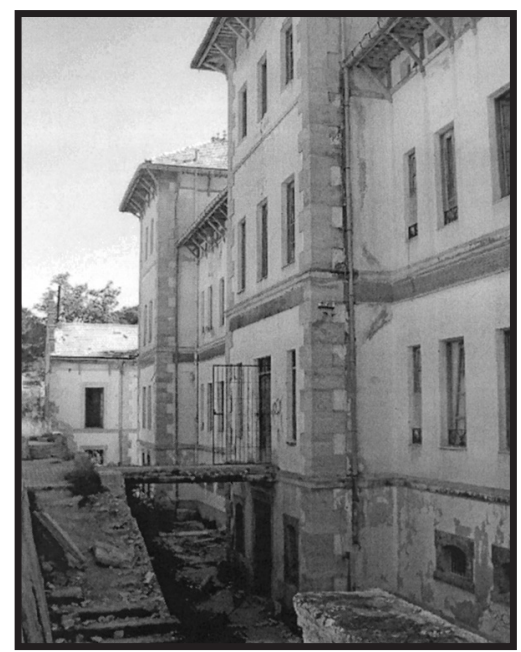

Vista del edificio principal y anexos

\section{BIBLIOGRAFÍA}

- AMEZCUA,M.: El Trabajo de Campo Etnográfico en Salud. Una aproximación a la observación participante.

- CASAS MARTÍNEZ,MF; RUIZ UREÑA,T; CARBELO BAQUERO,B; MIRALLES SANGRO,T; ROMERO LLORT,M.: (1998) Los cuidados de la enfermería en la infancia: El hospital del "Niño Jesús". Cultura de los cuidados, año II, n'III. Artículo de revista.

- DIPUTACIÓN DE ALICANTE.: Monumentos históricos-artísticos de Aigües.

www.dip-alicante.es/bonaigua/monumenai.htm.

- GABALDÓN BRAVO, EM; GÓMEZ ROBLES, FJ; FERNÁNDEZ MOLINA, MA; DOMINGO, M; TORRES FIGUEIRAS, M; TERRES RIUZ, C; GOMIS GARCÍA, L.: (2002) Análisis de las causas del bajo nivel de cumplimentación de la hoja de valoración del paciente al ingreso: una aproximación desde la perspectiva cualitativa. Cultura de los cuidados, año VI, $n^{\circ}$ XI, pag. 53-60. artículo de revista.

- JANSÁ LÓPEZ DEL VALLADO,JM; CAYLÁ IBUGUERAS, JA.: (2003) La tuberculosis, enfermedad clásica y emergente. Jano, 14-20 febrero 2003, nºXIV, pag,48-53. Artículo de revista.

- SILES GONZÁLEZ, J.: Epistemología y enfermería: por una fundamentación científica y profesional de la enfermería. Enfermería Clínica ,vol. VII, nº IV, pag 188-194. articulo especial.

- SILES GONZÁLEZ, J.: (2000) Antropología narrativa de los cuidados. Ed.: Consejo de Enfermería de la Comunidad Valenciana.

- SILES GONZÁlEZ, J.: (1999) Historia de la enfermería. Ed.: Aguaclara.

- SILES GONZÁLEZ, J.: (2001) Los textos como fuente de conocimientos en ciencias sociales y enfermería. Una aplicación de la metodología cualitativa. Index de Enfermería, X/34, pag.28-33.

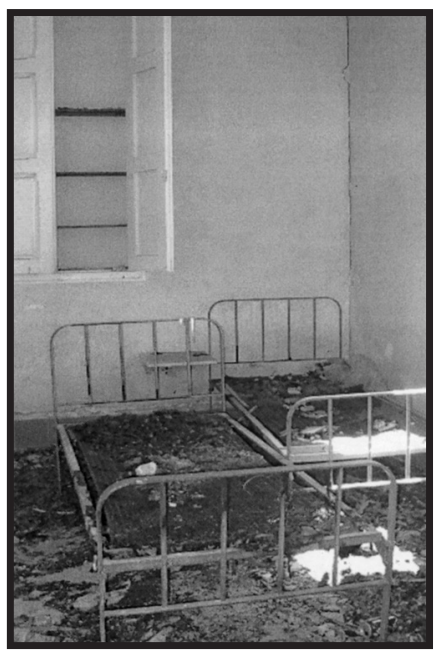

Situación de deterioro de las salas del sanatorio 


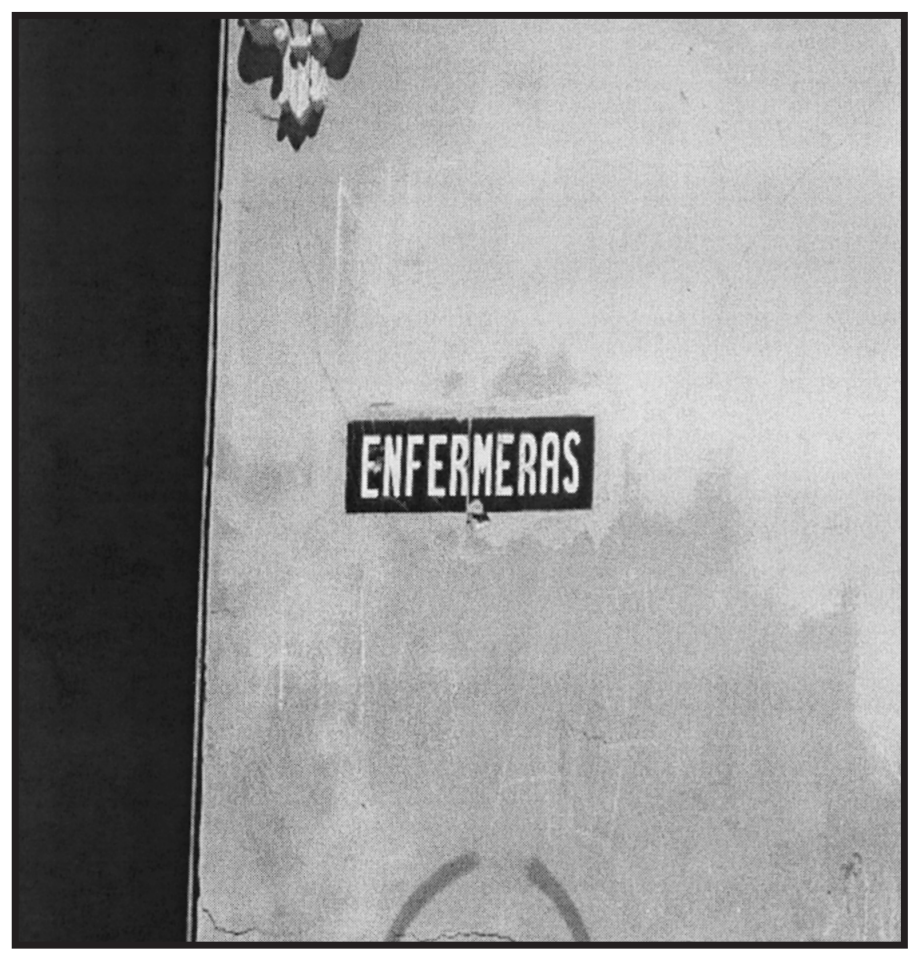

Tadavía se conserva, a la entrada de uno de los edificios anejos, el cartel que hace referencia a su uso por las enfermeras
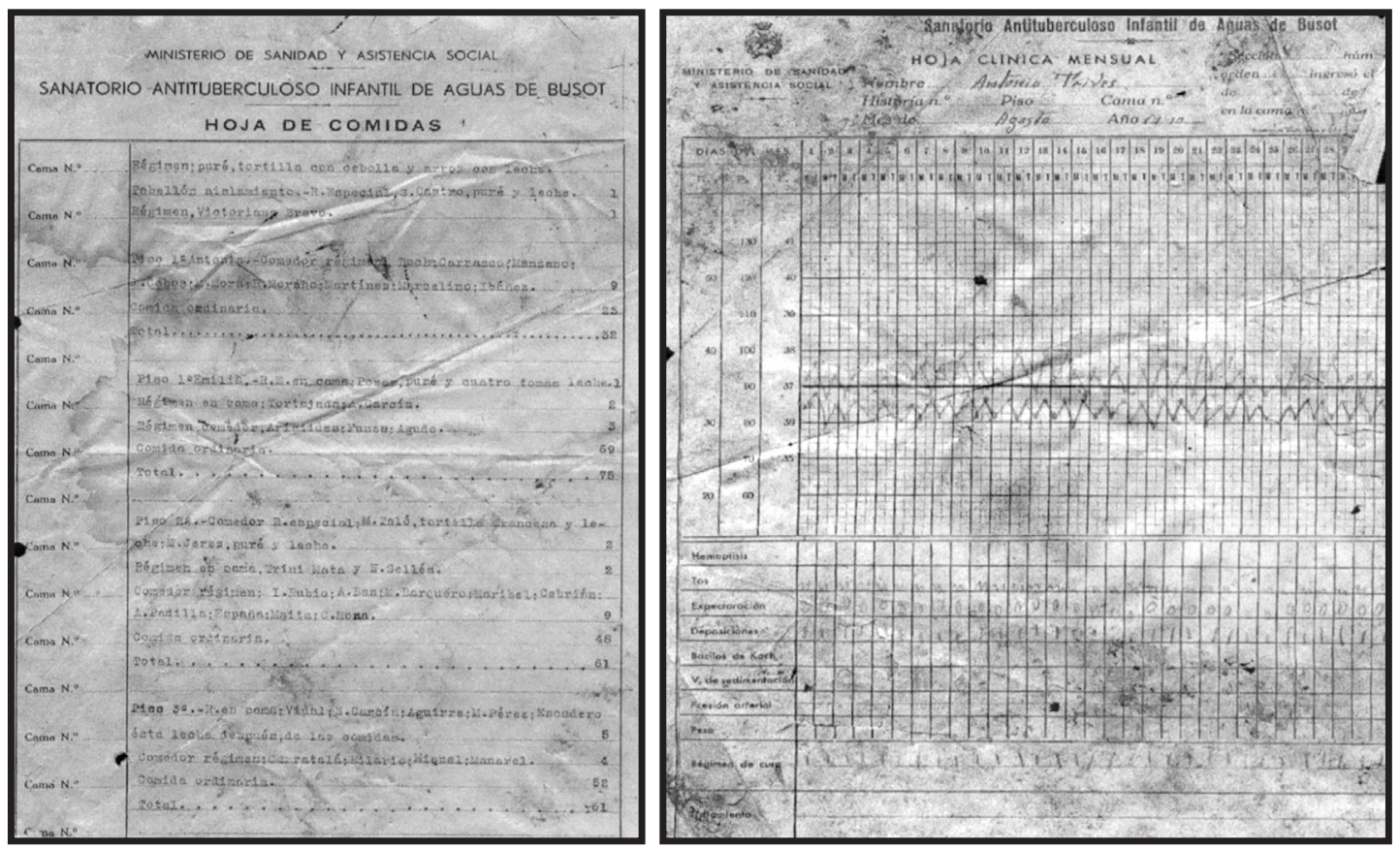

Distintos documentos rescatados 